\title{
Structure of Transfer Function of Transformers With Special Reference to Interleaved Windings
}

\author{
L. Satish and Anurag Jain
}

\begin{abstract}
Transfer function (TF) is computed during impulse tests on transformers. The structure and shape of TF depends on the type of winding, namely, disc, layer-type, or interleaved winding. There are certain features specific to TF of an interleaved winding, which has, over the years, been attributed to its increased series capacitance. However, reasons for why an increase in series capacitance should introduce such differences, the presence of only a few (lower frequency) poles in interleaved windings, etc., is as yet unknown. By analytically solving the equivalent circuit model, mathematical explanations are deduced. It is demonstrated, for the first time, how pole-zero cancellation and winding resistance are both instrumental in determining the structure of TF of interleaved windings.
\end{abstract}

Index Terms-Diagnostics, impulse tests, interleaved windings, transfer function (TF), transformers.

\section{INTRODUCTION}

$\mathbf{T}$ RANSFER FUNCTION (TF) is also computed from data acquired during high-voltage impulse tests on transformers. TF is defined as the ratio of spectra of neutral current to that of applied voltage. Use of TF affords many advantages over existing fault detection approaches and, in spite of some implementational and interpretation issues, has emerged as an effective diagnostic tool. TF is a graphical representation of the electrical characteristics of the winding as a function of frequency. The nature and shape of TF depends on the type of winding, knowledge of which is considered useful for diagnostics, and interpretation during impulse testing [1].

Generally, transformers have a higher likelihood of exposure to overvoltages. Furthermore, they are very expensive; therefore, it is imperative that adequate precautions are taken during the design stages itself. To withstand the stresses imposed due to these abnormal conditions, use of interleaved windings is recognized as an effective solution. Its superior surge performance due to improved impulse voltage distribution profile is the reason for its preferred choice, in place of disc or layer-type windings [2]. The main difference between these two winding types is a higher series capacitance in the former. A study of neutral currents and computed TFs (available in published literature) corresponding to these two types of windings show many differences. Until now, these differences have neither been systematically analyzed nor reasons for them ascertained. Before addressing this issue, salient features of their individual TFs are summarized as follows.

Manuscript received January 29, 2001.

L. Satish is with the Department of High Voltage Engineering, Indian Institute of Science, Bangalore 560012, India.

A. Jain is with Ramco Systems, Chennai, India.

Publisher Item Identifier S 0885-8977(02)05908-3.
1) $\mathrm{TF}$ of a layer-type winding has generally a number of poles that are more or less equally spaced, and typically occur within 0-1.0 MHz. All the poles are well defined with characteristic peaks and troughs. Pole magnitudes vary, but those at higher frequencies have progressively lower magnitude and tend to merge with noise floor.

2) In contrast, the TF of interleaved windings have only a few low-frequency poles, which occur well within $10 \mathrm{kHz}-300 \mathrm{kHz}$. The poles are spaced relatively more closely. The magnitude of higher frequency poles gradually decrease, and beyond the last pole, the TF tapers off into a straight line with a positive slope. There is a conspicuous absence of poles beyond $300 \mathrm{kHz}$, when a fully interleaved winding is examined alone [3]. However, often one encounters a single dominant pole in the $300 \mathrm{kHz}-1 \mathrm{MHz}$ zone, when TF is computed from actual impulse test data on an interleaved winding transformer. This pole is not a direct contribution of the interleaved winding, but perhaps due to an interaction with neighboring windings (explanation in support of this will be presented later). Because of this dominant pole, any fault related information embedded in the low-frequency poles will be suppressed, and can lead to erroneous interpretations. Therefore, a correct understanding of what governs the TF structure of interleaved windings would assist in avoiding such situations.

There have been many investigations on different aspects of interleaved windings (e.g., Heller and Veverka [2], Van Nuys [4], Pedersen [5], and Degeneff [6]). More specifically, in 1974, Gururaj and Jayaram [7] discussed the effect of change in series capacitance in a transformer on its neutral current. Furthermore, it was shown that only a large capacitance change can produce a perceptible deviation in the neutral current, implying that minor faults are hard to detect in an interleaved winding. More recently, Moreau et al. [8] while reporting on high-frequency behavior of transformers, attempted a comparison of interleaved and ordinary disk winding. Through simulations, they reported presence of a flat frequency region between 1 and $15 \mathrm{MHz}$, in interleaved windings. However, experiments on different transformers revealed a flat region in the $100-500 \mathrm{kHz}$ range. Without assigning any reasons for the mismatch, it was remarked that the flat zone could exist anywhere between $100 \mathrm{kHz}$ and $5 \mathrm{MHz}$. Actually, this is a consequence of an erroneous use of lumped equivalent circuit model at such high frequencies (in fact, some of them have been shown up to even $100 \mathrm{MHz}$ ).

In spite of many research efforts on interleaved windings, the fact remains that quantitative explanations for the peculiar TF structure of interleaved windings has, so far, remained unreported. Ascertaining reasons for this is not only interesting 


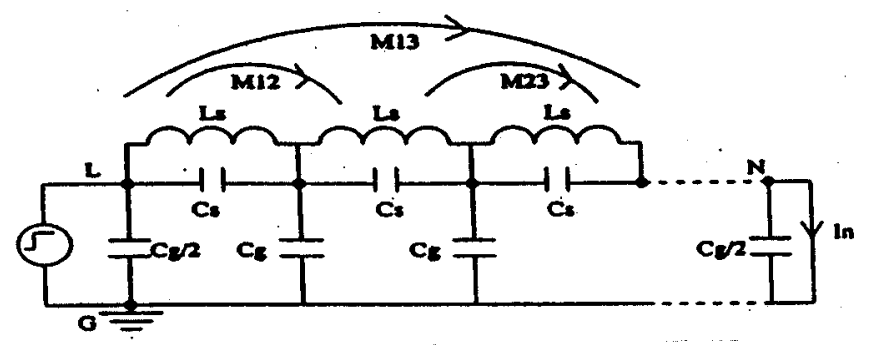

Fig. 1. Equivalent circuit.

from a theoretical standpoint, but more importantly, is expected to assist in arriving at a more meaningful interpretation of the TF, yield insight on precautions to be taken during data acquisition in impulse tests, and influence the diagnostic capabilities of transformers in service, especially when interleaved windings are used. These aspects have, to the best of our knowledge, not been investigated so far, and therefore form the subject matter of this paper.

\section{UNDERLYING APPROACH}

During high-voltage impulse testing and measurements, factors such as signal-to-noise ration (SNR), shape of applied impulse, digitizer vertical resolution, sampling frequency, and record length place an upper bound on the cutoff frequency up to which TF could reliably be estimated, to approximately 1.0-1.5 MHz. For this range of frequencies, the ladder network representation (see Fig. 1) comprised of series capacitance $C_{s}$, series inductance $L_{s}$, along with shunt capacitance $C_{g}$ and mutual inductance $M_{i-j}$, is known to accurately model all aspects of the neutral current response due to a lightning impulse $\left(C_{s}, C_{g}, L_{s}\right.$, and $M_{i-j}$ are per section values). As the validity of this circuit matches the frequency limit achievable during practical impulse measurements, it was considered appropriate to use it in the present context.

By using this circuit (see Fig. 1), the value of $C_{s}$ can be varied, and hence, the TF shape and structure corresponding to changes in $C_{s}$ can easily be tracked and examined. Both the layer-type and interleaved windings can easily be represented by changing $C_{s}$. This equivalent circuit, although not being exact for both these windings, is accurate enough as far as TF and neutral current are concerned. The equivalent circuit was analytically solved, for the following reasons.

1) At the outset, it must be emphasized that behavior of any system depends on its poles and zeros, its numbers and relative positions.

2) Analytical determination of poles and zeros not only reveals its exact number and magnitudes, but also the extent of dependencies on the equivalent circuit parameters.

3) Knowledge of poles and zeros as a function of the circuit elements greatly helps in tracking the change in the position of poles and zeros of the TF, and their likely interactions, if any.

4) It should be noted that determination of the poles and zeros numerically from test data is not straightforward, more so as it is a nonminimum phase system.
The equivalent circuit was solved using nodal analysis approach [9], [10]. Initially, a loss-less case was considered, and at the end, a resistance $r$ in series with $L_{s}$ was included. The neutral current expression $I_{n}(s)$ for a unit step input was determined. A unit step was chosen as input, instead of a double exponential, as it not only simplified computations, but also allowed determination of TF in analytical form, i.e., by dividing the $s$-domain neutral current expression by $1 / s$. The required symbolic computations were performed using MAPLE and MATLAB. As the focus was on behavior of TF with respect to $C_{s}$ and $C_{g}$, the neutral current expression was determined in terms of $C_{s}$ and $C_{g}$, by assuming typical values of series and mutual inductances. In all analytical computations, the neutral terminal was considered to be solidly grounded (i.e., with zero resistance). An examination of the neutral current for different number of sections $(n)$ in the equivalent circuit revealed that it could be represented by the following general format:

$$
\begin{array}{r}
I_{n}(s)=\frac{C_{s}^{n}}{\operatorname{poly}\left(C_{s}, C_{g}, m_{1}\right)}+\frac{K_{1}}{s^{2}}+\frac{\operatorname{poly}\left(C_{s}, C_{g}, s, m_{2}\right)}{\operatorname{poly}\left(C_{s}, C_{g}, s, m_{2}+2\right)} \\
-\frac{\operatorname{poly}\left(C_{s}, C_{g}, s, m_{3}\right)}{\operatorname{poly}\left(C_{s}, C_{g}, s, m_{3}+2\right)}
\end{array}
$$

where poly $(., .,$.$) represents a polynomial involving the vari-$ ables listed, the last term is its order, and the polynomial coefficients depend on $L_{s}$ and $M_{i-j}$. The polynomial order depends on the number of sections $n$, and when $n$ is odd, $m_{1}=n-1$, $m_{2}=m_{3}=n-3$, and when $n$ is even, $m_{1}=n-1$, $m_{2}=n-4$, and $m_{3}=n-2 . K_{1}$ is a constant, depending on $L_{s}$ and $M_{i-j}$. When values of $C_{s}$ and $C_{g}$ are plugged into the above equation, the first term turns out to be a constant and corresponds to the initial capacitive component of $I_{n}(s)$. The second term is a function of $s^{2}$ and corresponds to the inductive part of $I_{n}(s)$. The last two terms of the neutral current correspond to the oscillating components. Thus, the poles and zeros of the system can be determined, as a function of $C_{s}$ and $C_{g}$ from the last two terms. After some algebra, the poles and zeros of TF can be identified by inspection. As an example, consider a three-section model (see Fig. 1) with the following parameter values: $C_{s}=2 \mathrm{nF}, C_{g}=4 \mathrm{nF}, L_{s}=1 / 3 \mathrm{mH}$, $M_{1-2}=M_{2-3}=1 / 6 \mathrm{mH}$, and $M_{1-3}=1 / 12 \mathrm{mH}$. The analytically obtained neutral current is given below

$$
\begin{aligned}
I_{n}(s)= & \frac{C_{s}{ }^{3}}{3 C_{s}{ }^{2}+4 C_{s} C_{g}+C_{g}{ }^{2}}+\frac{6}{11 s^{2}} \\
& +\frac{1}{11} \frac{C_{s}^{2}+8 C_{s} C_{g}+16 C_{g}{ }^{2}}{\left(3 C_{s}+C_{g}\right)\left[s^{2}\left(3 C_{s}+C_{g}\right)+22\right]} \\
& -2 \frac{C_{g}{ }^{2}}{\left(C_{s}+C_{g}\right)\left[s^{2}\left(C_{s}+C_{g}\right)+4\right]} .
\end{aligned}
$$

The pole frequencies of $I_{n}(s)$ can be written down by inspection of its last two terms as

$$
f_{1}=\frac{1}{2 \pi \sqrt{\frac{\left(3 C_{s}+C_{g}\right)}{22}}}, f_{2}=\frac{1}{2 \pi \sqrt{\frac{\left(C_{s}+C_{g}\right)}{4}}} .
$$

Substituting the values (inductances are in millihenries and capacitances are in nanofarads; therefore, frequency obtained will be in megahertz), the natural frequencies are obtained as $f_{1}=$ 

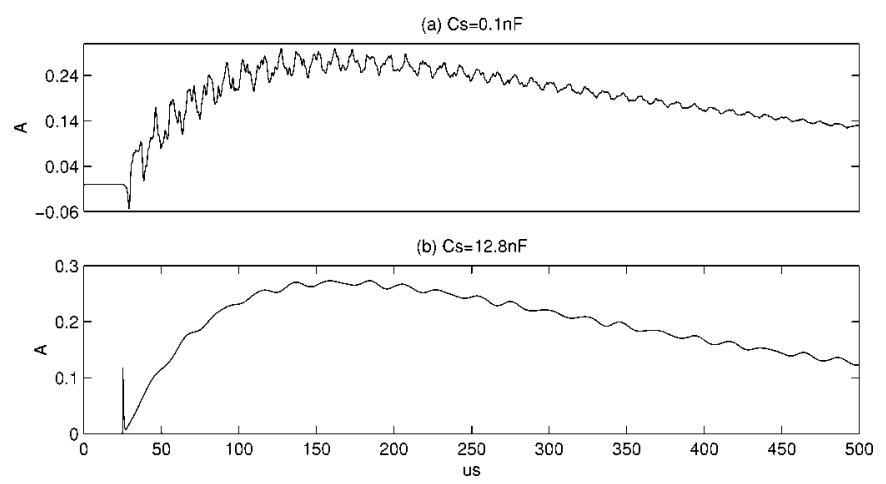

Fig. 2. Neutral currents. (a) Layer-type winding. (b) Interleaved winding.

$236.06 \mathrm{kHz}, f_{2}=129.95 \mathrm{kHz}$. Using values of $C_{s}$ and $C_{g}$, the neutral current expression becomes

$$
I_{n}(s)=\frac{2}{15}+\frac{6}{11 s^{2}}+\frac{81}{55\left(5 s^{2}+11\right)}-\frac{8}{3\left(3 s^{2}+2\right)} .
$$

These expressions of $I_{n}(s)$ and poles being analytical, are hence exact, and not subject to any limitations of sampling errors encountered in digital or sampled formulations. Finally, the similarity between $I_{n}(s)[(2),(4)]$ and the general expression of $I_{n}(s)$ in (1) is clear.

\section{RESULTS AND DISCUSSION}

In this paper, a ten-section equivalent network was considered. This circuit was analytically solved, and additionally, it was also simulated in PSPICE, so that TF could be computed from voltage and current time-domain records. Since, PSPICE does not permit inclusion of an ideal short-circuit, different values of neutral resistors were examined. As the final results were invariant to this change, a typical value of $50 \Omega$ was used in all PSPICE simulations to compute time-domain records. The other parameters of the circuit used are as follows.

$C_{g}=0.4 \mathrm{nF}, L_{s}=0.5 \mathrm{mH}$ and mutual inductances are $M_{1-2}=0.25 \mathrm{mH}, M_{1-3}=0.167 \mathrm{mH}, M_{1-4}=0.125 \mathrm{mH}$, $M_{1-5}=0.10 \mathrm{mH}, M_{1-6}=0.0833 \mathrm{mH}, M_{1-7}=0.0714 \mathrm{mH}$, $M_{1-8}=0.0625 \mathrm{mH}, M_{1-9}=0.0556 \mathrm{mH}, M_{1-10}=$ $0.050 \mathrm{mH}$, and $M_{1-2}=M_{2-3}=M_{3-4 \ldots}=M_{9-10}$, $M_{1-3}=M_{2-4}=M_{3-5 \ldots}=M_{8-10}, M_{1-4}=M_{2-5}=$ $M_{3-6 \ldots}=M_{7-10}$, and so on.

\section{A. Nature of Neutral Currents}

Using PSPICE, time-domain neutral current response due to a lightning impulse $1.2 / 50 \mu \mathrm{s}, V_{\text {peak }}=100 \mathrm{~V}$ (and computed at $50 \mathrm{~ns}$ time interval) was determined for both types of windings. Fig. 2(a) and (b) shows neutral current responses for two extreme values of $C_{s}$, namely, $0.1 \mathrm{nF}$ and $12.8 \mathrm{nF}$, which corresponds to a layer-type and interleaved winding, respectively, (corresponding $\alpha$ values are 20 and 1.77, respectively). It can be observed from Fig. 2(a) that, for the layer-type winding model considered, the neutral current response distinctly contains the intermediate oscillatory and final inductive components. In Fig. 2(b), for interleaved winding, the initial capacitive component is seen as a large positive spike, but the oscillating component is almost absent, and there is also a significant reduction in the frequency of oscillations. This aspect implies that TF of an interleaved winding would predominantly contain
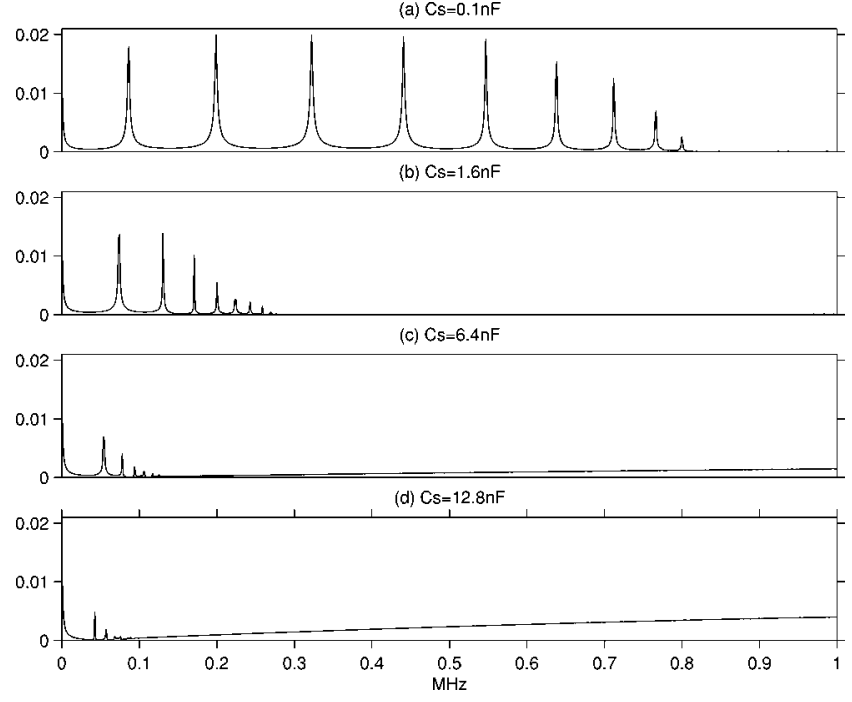

Fig. 3. TF from time-domain records for different values of $C_{s}$.

only lower natural frequencies. The series capacitance being very large in the case of interleaved winding, offers negligible impedance for the initial portion of the impulse, which causes a high spike (of same polarity of the impulse) in the neutral current. The corresponding spike in the layer-type winding is very small and immediately followed by the oscillatory part, and so, appears as a negative spike. Furthermore, shape of neutral current obtained from the model matches well with that measured on an untanked, fully interleaved winding (it was a fully interleaved winding taken out from a 110-kV, 40-MVA transformer. Its total $C_{g}$ was $3.44 \mathrm{nF}$ and value of $\alpha$ was about 4.0) [3]. This agreement further validates the model being considered in this analysis.

\section{B. TF Structure and its Variation With $C_{s}$}

TF computed from 16384 points long time-domain records (at $50 \mathrm{~ns}$ time interval) obtained from PSPICE for $C_{s}$ values of $0.1 \mathrm{nF}, 1.6 \mathrm{nF}, 6.4 \mathrm{nF}$, and $12.8 \mathrm{nF}$, for a 1.2/50 $\mu$ s applied impulse, are shown in Fig. 3. In all of these figures, the frequency resolution of TF is $1.22 \mathrm{kHz}$, and truncation in current and voltage records, if any, was removed by Nicolson weighting, to eliminate effect of spectral leakage on TF. From a study of Fig. 3, the following important observations emerge. As one moves from a layer-type winding to an interleaved winding, we have the following.

1) The number of poles in TF is significantly less.

2) All poles shift toward the lower frequency region.

3) As poles shift toward lower frequencies, they tend to come closer. The pole magnitudes decrease.

4) There is a conspicuous absence of poles beyond 90-100 kHz (this was anticipated during discussion in Section III-A).

5) After the last pole, the TF magnitude increases.

During this exercise of increasing the value of $C_{s}$, since the equivalent circuit topology had remained unchanged, one would normally have expected the circuit (and hence TF) to still continue to possess the same number of natural frequencies. However, in contrast, as the value of $C_{s}$ was increased, the TF computed from time-domain records was progressively found to contain a lesser number of poles. This aspect was indeed very 


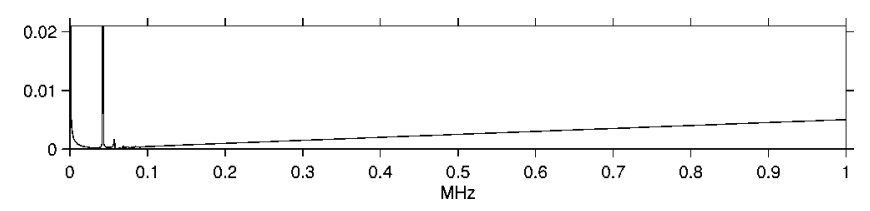

Fig. 4. Analytically obtained $\mathrm{TF}$ for $C_{s}=12.8 \mathrm{nF}$.

intriguing. In order to determine the reasons for such a behavior, analytical solution of the circuit was undertaken.

\section{Analytical Explanations for TF Behavior}

The expression for $I_{n}(s)$ was determined for a unit step input. Because of this, poles and zeros of TF will be the same as poles and zeros of $I_{n}(s)$, except that the TF will have an additional zero at the origin [because $T F=s^{*} I_{n}(s)$ ]. Therefore, by examining the poles and zeros of $I_{n}(s)$, behavior of TF can be explained.

The analytically obtained TF was plotted for the same values of $C_{s}$, retaining the same frequency resolution of $1.22 \mathrm{kHz}$. However, no significant differences in TF were observed up to $1 \mathrm{MHz}$. For example, Fig. 4 shows analytically computed TF for $C_{s}=12.8 \mathrm{nF}$. At higher frequencies, the analytically computed TF magnitude was observed to continuously increase. Such an increase of TF beyond the last pole is not surprising, as TF is governed by only the capacitive term, as per (1). A saturation-like feature in TFs computed from time-domain records at very high frequencies is due to sampling limitations. Analytically computed TFs do not suffer from this issue.

Next, the pole frequencies of $I_{n}(s)$ were analytically computed. These values, and those obtained from time-domain records, could more easily be matched, in the case of lower value of $C_{s}$ where the poles are easy to identify in TF plots. However, at higher $C_{s}$ values, as the poles tend to merge, discriminating between them is increasingly difficult. To address this issue, the record length was artificially increased by zero-padding. Merely increasing the record length by zero padding does not lead to any additional signal content, and therefore, it did not improve the situation. When the poles of $I_{n}(s)$ were determined analytically, it was found that all the pole frequencies (a ten-section network will have nine natural frequencies) could easily be computed, irrespective of the value of $C_{s}$ used. However, that was not the case when TF was computed from time-domain records, as discussed previously. This issue is considered next.

It is known that the behavior of any system is governed by both the poles and zeros, their relative numbers, and relative spatial positions. Until now, investigations have concentrated only on analyzing the system poles and the role of zeros seems to have been ignored. Therefore, keeping this aspect in mind, it was decided to analytically determine the zeros as well. Unlike the expression for poles, the equations for zeros were relatively longer and more complex, and so, nothing worthwhile could be inferred based on mere inspection. Therefore, by plugging-in values of all the parameters, poles and zeros of $I_{n}(s)$ were numerically determined. These values were determined for all $C_{s}$ values and shown in Table I for two extreme values of $C_{s}$. The number of zeros is one more than the number of poles, and the zero that is closest to a particular pole is paired together. Fig. 5 is a pole-zero plot for all values of $C_{s}$ to facilitate easy interpre-
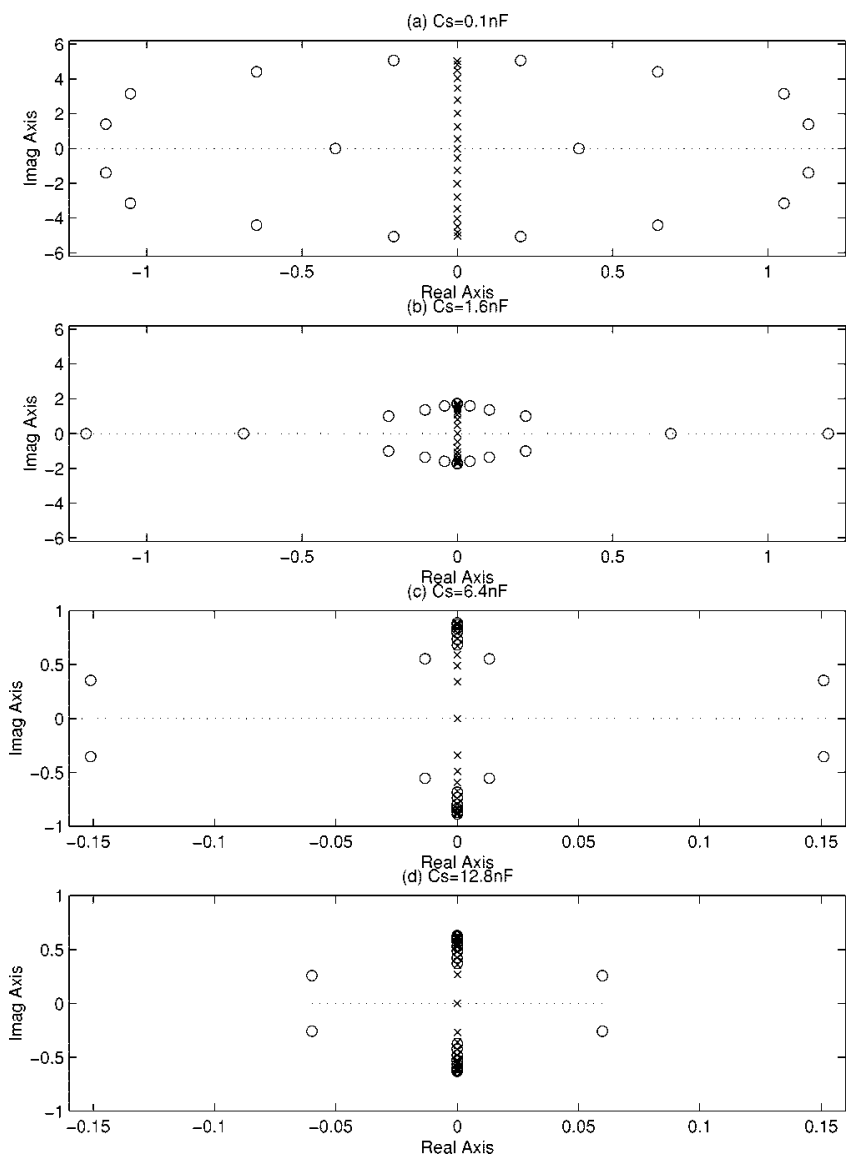

Fig. 5. Pole-zero plots of $I_{n}(s)$ for different values of $C_{s}$.

TABLE I

(Mega-Neper $\pm j$ Mega-rad/s)

\begin{tabular}{l|c|c}
\hline$\#$ & POLE & ZERO \\
\hline 1 & $0 \pm 0.541314676 \mathrm{i}$ & \multicolumn{2}{c}{$\mathrm{C}_{s}=0.1 \mathrm{nF}$} \\
\hline 2 & $0 \pm 1.250909968 \mathrm{i}$ & $1.131510356 \pm 1.393517878 \mathrm{i}$ \\
\hline 3 & $0 \pm 2.029155244 \mathrm{i}$ & $-1.131510356 \pm 1.393517878 \mathrm{i}$ \\
\hline 4 & $0 \pm 2.783836479 \mathrm{i}$ & $1.052698218 \pm 3.151536121 \mathrm{i}$ \\
\hline 5 & $0 \pm 3.465691379 \mathrm{i}$ & $-1.052698218 \pm 3.151536121 \mathrm{i}$ \\
\hline 6 & $0 \pm 4.040554368 \mathrm{i}$ & $0.645839974 \pm 4.408211845 \mathrm{i}$ \\
\hline 7 & $0 \pm 4.496358158 \mathrm{i}$ & $-0.645839974 \pm 4.408211845 \mathrm{i}$ \\
\hline 8 & $0 \pm 4.824173487 \mathrm{i}$ & $0.204254895 \pm 5.066090986 \mathrm{i}$ \\
\hline 9 & $0 \pm 5.021941125 \mathrm{i}$ & $-0.204254895 \pm 5.066090986 \mathrm{i}$ \\
\hline 10 & 0 & \pm 1766.433050 \\
\hline 1 & $0 \pm 0.268324663 \mathrm{i}$ & $0.059863124 \pm 0.257793995 \mathrm{i}$ \\
\hline 2 & $0 \pm 0.359040788 \mathrm{i}$ & $0 \pm 0.372279781 \mathrm{i}$ \\
\hline 3 & $0 \pm 0.426686939 \mathrm{i}$ & $0 \pm 0.422581721 \mathrm{i}$ \\
\hline 4 & $0 \pm 0.480914853 \mathrm{i}$ & $0 \pm 0.483438021 \mathrm{i}$ \\
\hline 5 & $0 \pm 0.527409684 \mathrm{i}$ & $0 \pm 0.526572716$ \\
\hline 6 & $0 \pm 0.565037440 \mathrm{i}$ & $0 \pm 0.565689780 \mathrm{i}$ \\
\hline 7 & $0 \pm 0.594909141 \mathrm{i}$ & $0 \pm 0.594714918 \mathrm{i}$ \\
\hline 8 & $0 \pm 0.616246540 \mathrm{i}$ & $0 \pm 0.616370812 \mathrm{i}$ \\
\hline 9 & $0 \pm 0.629202466 \mathrm{i}$ & $0 \pm 0.629184618 \mathrm{i}$ \\
\hline 10 & 0 & $-0.059863124 \pm 0.257793995 \mathrm{i}$ \\
\hline & &
\end{tabular}

tation. From an examination of Fig. 5 and numerical values in Table I, the following important points emerge.

1) All poles lie on the imaginary axis (as they should). 


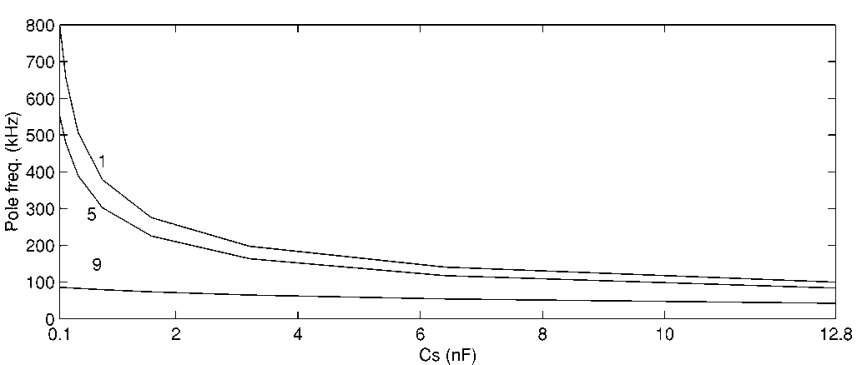

Fig. 6. Variation of pole frequencies with $C_{s}$.

2) An increase of $C_{s}$ causes poles to move along the imaginary axis toward the origin.

3) Zeros are also present on the right-half plane, showing that it is a nonminimum phase network. Zeros and poles are well separated at lower values of $C_{s}$. A symmetry is observed about the real axis (as expected) and imaginary axis as well.

4) An increase of $C_{s}$ causes zeros to rapidly migrate toward and settle on the imaginary axis and thereby come closer to the poles.

5) At higher values of $C_{s}$, most of the poles and zeros are almost indistinguishable. For example, at $C_{s}=12.8 \mathrm{nF}$, it is seen in Table I, that the higher frequency pole and zero frequencies differ by as little as only a few tens of hertz. Such a small difference cannot be resolved in the pole-zero plot, since they overlap. In the TF, these particular pole-zero pairs almost cancel out, leaving that many fewer natural frequencies, and reduce the order of the system. This is what is usually referred to as a pole-zero cancellation.

6) The pole-zero cancellation is observed to first occur for the higher frequency poles, and then, gradually affect to the lower frequency poles, as $C_{s}$ is increased.

Therefore, it is now clear that at higher values of $C_{s}$, i.e., in an interleaved winding, because of pole-zero cancellation, the TF will effectively contain only a few distinct low-frequency poles. As the difference between the pole and zero is of the order of a few tens of hertz, it is certain that, when using time-domain records most of these poles will remain undetected. Therefore, efforts must be made to keep the frequency resolution close to this limit, if all poles are to be resolved. This is an uphill task, since it means that a record length of close to $10^{5}$ points has to be acquired. This is practically not possible in many of the existing impulse measuring systems. Therefore, this situation suggests employment of an alternative, e.g., the swept frequency method. Before discussing that issue, another feature observed in TF, namely, the tendency of the natural frequencies to shift toward the lower frequency region (when $C_{s}$ is increased), was studied, by examining the dependency of pole frequencies on $C_{s}$. Substituting values of all the parameters into the expression, pole frequencies (pole numbers 1,5 , and 9 from Table I) as a function of $C_{s}$, are plotted in Fig. 6, from which the following observations can be made.

1) Pole frequencies monotonically decrease with an increase in the value of $C_{s}$, i.e., they shift toward low-frequency region.

2) The higher frequency poles shift more rapidly compared to low-frequency poles.

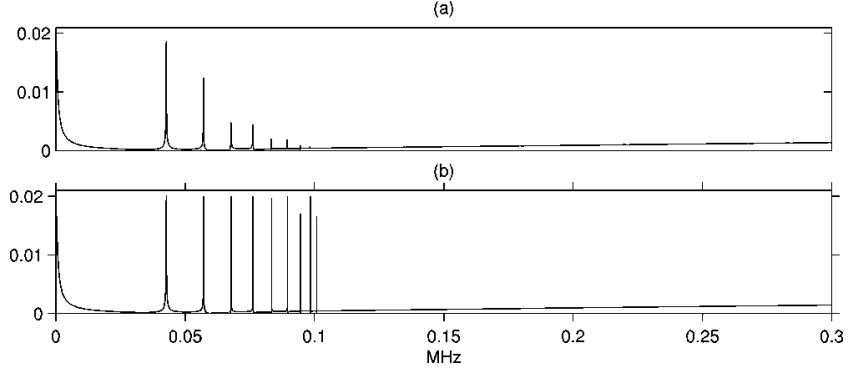

Fig. 7. $\mathrm{TF}$ for $C_{s}=12.8 \mathrm{nF}$. (a). Using long time-domain records (frequency resolution $=122.07 \mathrm{~Hz})(\mathrm{b})$. Using swept frequency method (frequency step $=1 \mathrm{~Hz}$ ).

3) The pole frequencies reach a limiting value beyond a particular $C_{s}$ value (this is also seen in Fig. 5).

An attempt was also made to describe this feature, by means of a relation between pole frequency and $C_{s}$. It turned out that the expressions were extremely long (running to pages) and approximating them was not possible, nor could any inference be drawn from it. Hence, only a qualitative assessment was done.

\section{Swept Frequency Methods}

As already discussed, in actual impulse measurements, the minimum attainable frequency resolution in $\mathrm{TF}$ is of the order of a few kilohertz. This feature becomes a bottleneck when analyzing the test data of transformers with interleaved windings, since extremely small frequency resolutions (of the order of a few tens of hertz) are necessary to resolve all the natural frequencies present, to ensure reliable diagnostics and correct interpretation of TF.

It is reiterated that zero-padding an existing $4 \mathrm{~K}$ or $8 \mathrm{~K}$ long time-domain record, will not produce the desired results, because doing so, does not introduce any additional information content into the signal. Instead, the other possibility is to acquire the time-domain data for extended lengths, and by doing so, definitely additional low-frequency information gets included. In such a situation, an improvement of resolution at lower frequencies could be expected. With $C_{s}=12.8 \mathrm{nF}$, the voltage $(1.2 / 50 \mu \mathrm{s})$ and neutral current were calculated at $200 \mathrm{~ns}$ for 40960 points, yielding a TF frequency resolution of $122.07 \mathrm{~Hz}$. TF calculated with these time-domain signals is shown in Fig. 7(a). Clearly, four poles can be identified, which is an improvement compared to Fig. 3(d), but all nine poles could not be resolved. Increasing the record length further was not found to be very effective.

A practical way of achieving such small frequency resolution is to explore the use of swept frequency methods. Theoretically, this option can be implemented in PSPICE using the option "LIN AC SWEEP ANALYSIS." Choosing a frequency step of $1 \mathrm{~Hz}$, the computation was performed (for $C_{s}=12.8 \mathrm{nF}$ ), and the resulting TF is shown in Fig. 7(b). It is clear that all nine poles can be identified, even when $C_{s}=12.8 \mathrm{nF}$. The analysis with $1 \mathrm{~Hz}$ resolution can be restricted up to 100 or $150 \mathrm{kHz}$ to limit data size.

Furthermore, a closer look at the TF obtained in Fig. 7(b) shows that, beyond the first few low-frequency poles, the rest of them are all extremely sharp (loss-less case) and have a peculiar shape near their troughs. A zoomed TF plot showing this aspect is illustrated in Fig. 8. Such a TF shape near the troughs 


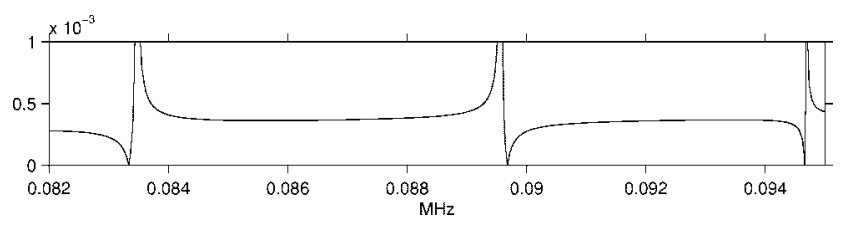

Fig. 8. Zoomed plot of TF in Fig. 7(b).

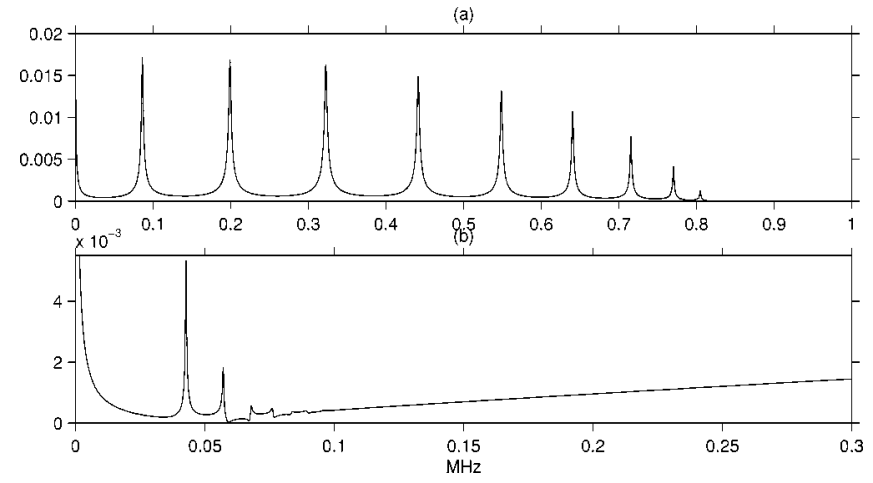

Fig. 9. Swept frequency analysis with loss considered. (a). $C_{s}=0.1 \mathrm{nF}$ (frequency step $=50 \mathrm{~Hz}$ ). (b). $C_{s}=12.8 \mathrm{nF}$ (frequency step $=1 \mathrm{~Hz}$ ).

is due to the existence of a near-discontinuity in the TF expression, and occurs at every pole-zero cancellation. This is a feature in all TFs, where the poles and zeros are extremely close to one another, almost resulting in a cancellation. A physical implementation of the idea of swept frequency method is possible with the use of network analyzers or low-frequency impedance analyzers. Such instruments are presently being used for FRA analysis in diagnosing mechanical deformations in transformer windings.

\section{E. Effect of Loss}

Although pole-zero cancellation is the primary cause for a lesser number of poles in the TF of an interleaved winding, the reason for studying the effect of loss is that the quality factor and pole height in the TF depends on the loss (resistance) in the system. Therefore, inclusion of a loss component into the simulation or equivalent circuit can definitely be expected to have an influence on the results obtained so far.

With a resistance of $1.5 \Omega$ included in series with $L_{s}$ in each section and a $50 \Omega$ neutral resistance, a PSPICE simulation was performed (LIN AC SWEEP ANALYSIS) for two extreme values of $C_{s}(0.1 \mathrm{nF}$ and $12.8 \mathrm{nF})$. TF obtained for $C_{s}=0.1 \mathrm{nF}$ with a frequency step of $50 \mathrm{~Hz}$ is shown in Fig. 9(a), and it can be seen that all the poles are well resolved.

Of course, in a layer-type winding, this has never been an issue. In Fig. 9(b), TF computed for $C_{s}=12.8 \mathrm{nF}$, with a frequency step of $1 \mathrm{~Hz}$ is plotted. Surprisingly, only two poles can be identified. Comparing this TF with that in Fig. 7(b), it can be seen that pole heights have significantly decreased and, in addition, there is a widening of the poles, i.e., their $q$-factors have increased. Thus, in an interleaved winding, pole widening and reduction in pole height due to losses, are two more reasons the remaining higher frequency poles cannot be resolved or detected, irrespective of the adopted approach.

To prove this aspect, the three-section network model described in Section II, is again considered, but with loss included (i.e., a resistance $r$ in series with $L_{s}$ ) and neutral

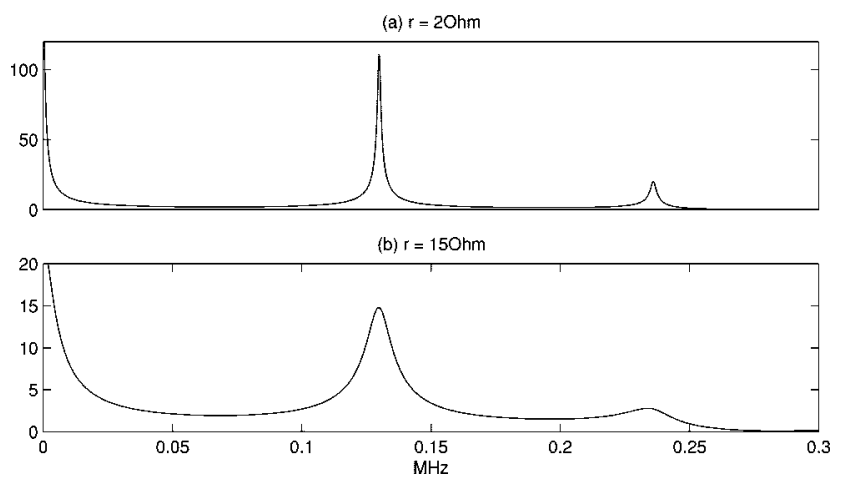

Fig. 10. Analytically obtained TF for different values of $r$.

solidly grounded. It was solved analytically, and the expression for TF in terms of $r$ and $s$ is

$$
\begin{aligned}
T F= & \frac{N 1}{D 1} \\
N 1= & 2 s^{6}+(26 r) s^{5}+\left(96 r^{2}+5\right) s^{4}+\left(96 r^{3}+32 r\right) s^{3} \\
& +\left(144 r^{2}\right) s^{2}+(72 r) s+12 \\
D 1= & 15 s^{5}+(195 r) s^{4}+\left(720 r^{2}+43\right) s^{3}+\left(720 r^{3}+276 r\right) s^{2} \\
& +\left(336 r^{2}+22\right) s+36 r .
\end{aligned}
$$

Here, $C_{s}$ and $C_{g}$ are in nanofarads, $L_{s}$ is in millihenries, and $r$ is in kilohms. TF plotted for $r=2 \Omega$ and $15 \Omega$ is shown in Fig. 10. An increase in $r$ leads to a drastic reduction in pole height and width of the pole also increases. It is seen that the second pole is almost buried in the background. In interleaved windings, since magnitudes of higher frequency poles are low to begin with, an inclusion of loss will further decrease it, making its detection all the more difficult. In addition, the factor of pole widening due to losses, makes it even more difficult to resolve them, especially with neighboring poles being very close to one another. Because of this, they tend to merge together, and appear as a single pole, or may remain undetected. Thus, resistance or loss of the network also plays a vital role in modifying the shape of TF.

\section{F. Implications on Diagnostics}

As already discussed in Section I, difficulties connected with fault detection in interleaved windings were highlighted in [7]. Based on the present results, certain guidelines can be formulated so that interpretations and diagnostics with TF, for interleaved windings in particular, could be improved.

1) It is advisable to employ swept frequency methods to measure TF directly, using low-frequency impedance analyzers (e.g., HP 4192A). For this, only the low-frequency region $(10 \mathrm{kHz}-100 \mathrm{kHz})$ of the TF needs to be scanned. Use of frequency steps of the order of a few tens of hertz is mandatory. This method is only an additional diagnostic tool.

2) Ascertaining dielectric integrity can only be done by HV impulse testing. However, if a few suggestions given below can be adhered to while acquiring impulse test data, then, the TF estimated with it will be closer to the TF obtained from swept frequency method. Therefore, during impulse tests, it is recommended that a maximum possible length of the signals should be recorded. For this, even a coarse sampling time can be used, as 
high-frequency content in the signal is absent. Data must be Nicolson-windowed prior to TF estimation. During TF interpretation, any dominant high-frequency pole (arising from an interaction with neighboring windings) must be disregarded, and attention focused only on the $0-300 \mathrm{kHz}$ region alone. This rule is also true for detecting mechanical displacements, since physical changes in windings are most likely to introduce deviations in $\mathrm{TF}$ within this range. Attention of the reader is drawn here, to the fact that the TF of interleaved windings discussed so far did not contain any poles beyond the $200-\mathrm{kHz}$ region. Also, TF computed in [3] on a fully interleaved winding did not possess any pole beyond $250 \mathrm{kHz}$. Therefore, if the $\mathrm{TF}$, computed from actual impulse test data, possesses a pole in the range of $200 \mathrm{kHz}-1 \mathrm{MHz}$, its origin can only be traced to its interaction with neighboring windings.

3) Given that interleaved windings have TF information predominantly concentrated in the low-frequency region, a question that needs to be answered is, would it would be prudent to employ a much slower impulse waveform for purposes of estimating the TF? This would excite lower frequencies better, thereby improving the SNR. This suggestion is purely from the LV diagnostics point of view, and does not intend to replace the LI test. An answer to this can emerge, only after relevant studies are carried out.

It is important to mention at this juncture, that some experiments have been carried out on a model transformer winding. The preliminary results obtained using swept frequency method agree well with those predicted and reported here. Further experimentation and verification is currently underway, and details of these will be reported later.

Finally, in summary, the following important results emerge from this study.

$\triangleright$ In an interleaved winding, a situation similar to a pole-zero cancellation almost occurs. Due to this, its TF effectively contains only a few distinct low-frequency poles.

$\triangleright \mathrm{TF}$ of an interleaved winding when considered alone, beyond the last pole, is a straight line with a positive slope. $\triangleright$ Loss in the network causes a reduction in pole height and also introduces a pole widening. These two factors complicate matter further as resolution of the remaining poles becomes almost impossible. Use of swept frequency methods (with frequency steps of the order of a few hertz) for estimating the TF of interleaved windings was shown to be effective.

$\triangleright$ During HV impulse tests (involving interleaved winding transformers), it would be beneficial to acquire test data for extended lengths, if permissible by hardware, for improving resolution of low-frequency poles. The TF estimated with such data would be closer to the true TF that could be estimated by swept frequency methods.

$\triangleright$ Proposed use of slower front impulses for LV diagnostics.

\section{CONCLUSIONS}

Differences in TF between a layer-type and interleaved winding were examined. Mathematical explanations have been provided, for the first time, to explain the peculiar TF structure observed in interleaved windings. It was demonstrated both by numerical and analytical approaches, how pole-zero cancellation and network loss are together instrumental in determining the structure of TF. Some suggestions for data acquisition during impulse tests on interleaved winding transformers are given, so that a more accurate TF could be estimated. The advantages of using swept frequency methods to directly measure $\mathrm{TF}$ was shown. It is believed that, based on the present findings, there definitely exists a scope for improving diagnostics with $\mathrm{TF}$, especially for transformers with interleaved windings.

\section{ACKNOWLEDGMENT}

The authors thank Prof. B. I. Gururaj for suggesting this very interesting problem. The authors have benefited from his remarks and criticisms. His suggestions during preparation of the paper are sincerely acknowledged.

\section{REFERENCES}

[1] R. Malewski and B. Poulin, "Impulse testing of power transformers using the transfer function method," IEEE Trans. Power Delivery, vol. 3, pp. 476-489, Apr. 1988.

[2] B. Heller and A. Veverka, Surge Phenomena in Electrical Machines. London, U.K.: Iliffe Books, 1968.

[3] M. L. Pykala, V. Palva, J. Hallstrom, M. Aro, and L. Satish, "Transfer function of power transformer-Part IV," Helsinki University of Technology, Helsinki, Finland, High Voltage Institute Rep., TKK-Sjt-36, 1999.

[4] R. van Nuys, "Interleaved high-voltage transformer windings," IEEE Trans. Power App. Syst., vol. PAS-97, pp. 1946-1954, Apr. 1978.

[5] A. Pedersen, "On the response of interleaved transformer windings to surge voltages," IEEE Trans. Power App. Syst., vol. PAS-66, pp. 349-356, June 1963.

[6] R. C. Degeneff, "A general method for determining resonances in transformer windings," IEEE Trans. Power App. Syst., vol. PAS-96, pp. 423-430, Mar. 1977.

[7] B. I. Gururaj and B. N. Jayaram, "Use of recurrent surge generator for detecting displacements and deformations in transformer windings," $J$. Inst. Eng, (India), vol. 54, no. EI-3, pp. 99-102, 1974.

[8] O. Moreau, P. Guuinic, R. Dorr, and Q. Su, "Comparison between the high-frequency characteristics of transformer interleaved and ordinary disk windings," in Proc. IEEE PES Winter Meeting, vol. 3, Singapore, Jan. 2000, pp. 2187-2192.

[9] E. A. Guillemin, Introductory Circuit Theory. New York: Wiley, 1953.

[10] A. Jain, "Structure of transfer function of transformers with special reference to interleaved windings," M.S. thesis, Dept. High-Voltage Eng., Indian Institute of Science, Bangalore, India, Mar. 2001

L. Satish was born in 1964. He received the Ph.D. degree from the Indian Institute of Science (IISc), Bangalore, in 1993.

He was a Postdoctoral Fellow at ETH, Zurich, Switzerland, from 1993 to 1995. In 1995, he joined the Department of High-Voltage Engineering, Indian Institute of Science, where he is currently an Associate Professor. His research areas include application of signal processing to $\mathrm{HV}$ impulse testing and diagnostics, partial discharge measurements, and pattern recognition. He was a Visiting Researcher at the High-Voltage Institute, Helsinki University of Technology, Helsinki, Finland, during the summer of 1998.

Dr. Satish is a Member of TF 1.1 of the CIGRÉ WG 33-03.

Anurag Jain was born in 1978. He received the B.Tech. degree in electrical engineering from Harcourt Butler Technological Institute (HBTI), Kanpur, India, in 1999, and the M.Sc. degree in engineering from the Department of High-Voltage Engineering, Indian Institute of Science, Bangalore, in 2001. His research topic was the behavior of transfer functions of different types of transformer windings.

He is currently a Research Engineer with Ramco Systems, Chennai, India. His current research interests are signal processing and its use for diagnostics to transformers. 